\title{
Overview and challenges of palm vein biometric system
}

\begin{abstract}
Palm vein biometric system is one of the biometric technologies that has grabbed the attention of scholarly researchers and industrial alike, due to its distinctive properties and hidden nature. Constant effort had been done in improving the palm vein biometric system performance through the design of its vein acquisition system and vein image analysis. This paper provides an overview of the underlying elements of a palm vein biometric system that summarises the works done, and predicts the upcoming research focus in this area.
\end{abstract}

Keyword: Palm vein biometrics; Palm vein patterns; Image analysis; Performance evaluation; Image acquisition; Biometric recognition systems 